\title{
PROBABILITY OF BOUNDARY EXHAUSTION OF RESOURCES AS FACTOR OF OPERATIONAL SAFETY FOR AGRICULTURAL AGGREGATES
}

\author{
Georgiy Pisarenko ${ }^{1}$, Oleksandr Voinalovych ${ }^{2}$, Ivan Rogovskii ${ }^{2}$, Myhailo Motrich ${ }^{2}$ \\ ${ }^{1}$ Institute for Problems of Strength National Academy of Sciences of Ukraine, Ukraine; \\ ${ }^{2}$ National University of Life and Environmental Sciences of Ukraine, Ukraine \\ pysarenko@nas.gov.ua, voynalov@bigmir.net,rogovskii@nubip.edu.ua,mmi@online.ua
}

\begin{abstract}
The influence of the presence of fatigue cracks detected in the array of operational defects of typical parts of individual units on the condition of safety of tractors has been analyzed. In the work with the help of a specialized portable vibration current flow defectoscope the presence of cracks in the details of the units of tractors with different operating periods was estimated. The defectoscopic control of more than 500 parts of various applications was carried out in 50 tractors that were in service until 17 years since the date of issue in agricultural enterprises of Ukraine. Defectoscopic inspection was carried out during the defect of the details of these tractors received for repair. Based on statistically valid data, the kinetic dependences of the probability distribution of the accumulation of operational fractures of parts of tractor units in time were constructed. The cracks analyzed in the work were detected using a high-sensitivity pulsed vibration current flaw detector, which allowed us to quantify the size of the cracks that correspond to certain operating lifetime terms. It is established that the process of accumulation of a certain type of cracks has exponential kinetics. The regularities considered in the work reflect the consequences of the statistical process of accumulation of an array of fatigue cracks in the details (knots) of tractors within the limits of the studied range of service life. Exceeding the maximum period of operation of aggregates affects the change in the probability of accidents in mechanized and transport works. It is determined that the deadline for the researched tractor types is 13-14 years.
\end{abstract}

Keywords: crack, defectoscopic control, tractor, aggregate, resource.

\section{Introduction}

The high level of injuries in the agroindustrial complex is due to the fact that most of the technological operations are carried out using morally obsolete and used equipment [1]. However, researchers of occupational injuries in the agroindustrial complex primarily focus on organizational reasons [2] and working conditions [3], largely ignoring the unsatisfactory technical condition of the mobile means of agribusiness production.

At present, in the agroindustrial complex, in the face of high depreciation wear on the wheel equipment, it is not fully possible to solve the issue of improving the safety of machine operators and drivers [4]. Studies on the setting of conditions for and the safety of work on tractors, harvesters or trucks generally belong to secondary [5].

The analysis of the research in professional risk assessment in the agroindustrial complex showed that the used methods today largely do not determine the quantitative characteristics of the safety of the system of human-machine-environment (H-M-E) and can be used as an estimate of individual indicators [6]. On the other hand, in works dealing with the functioning of the mathematical model of the system H-M-E in agricultural production, the main emphasis is mainly on the reliability aspects of mobile technology [7]. But at the same time they do not consider the potential hazards for machine operators and other workers involved in the production process.

Thus, controlling the quality of maintenance and repair of mobile agricultural machinery, for the most part they pay attention to the definition of technological and economic performance of the machine, without monitoring the existence of operational defects (cracks) in details. And this often leads to the implementation of mechanized work with an increased level of accidents, which leads to traumas of mechanics and auxiliary workers [8].

The comparison of such lines of analysis of the system H-M-E, as the reliability of equipment and labor protection during its operation, shows that they are based on the same probabilistic models of risk assessment failures and, respectively, the onset of accidents [9]. Therefore, in order to assess the probability of occurrence of emergencies it is expedient to involve approaches of the reliability theory, based on the statistics of failures of machinery or equipment.

The analysis of literary sources allows us to put forward the hypothesis that the statistical data of the defectoscopic control of the existence of operational cracks in the array of parts of the individual 
nodes determining the safety of the operation of the tractors can be used to establish the patterns of resource depletion, prediction of reliability and safety factors of long-life aggregates [10].

Obtaining of statistically substantiated data of the defectoscopic control during maintenance and repair of agricultural machinery constrains the non-adaptability of portable defectoscopes for the operational diagnosis of individual parts of tractors and self-propelled agricultural machines (SAM). In particular, there are currently no records of operational defects of parts of mobile machinery units after prolonged use. The purpose of the study is to substantiate the deadlines for the operation of mobile agricultural machinery with a high degree of damage to parts.

To achieve this goal you need to solve the following tasks.

1. Analyze the possibilities of adaptation of portable defectoscopes for operative detection of operational damage in an array of parts units of tractors and SAM.

2. Obtain and analyze the kinetic dependences of the accumulation of defects in the details of the knots of tractors of different durations of operation.

The basis of the research was the thesis that the probability of failure of individual tractor units is determined by a complex of existing cracks in details, which imposes special requirements for the periodicity and diligence of the conduct of defectoscopic control. At the same time, the reliability of the forecast depends on the quality of the received information on the presence of defects in the responsible details and elements of the structures of such objects. The proposed approach does not contradict the methods of statistical estimation of the probability of failure-free operation of agricultural machinery, developed by other authors $[4 ; 6 ; 7]$.

\section{Materials and methods}

In the work with the help of a specialized portable vibration current flow defectoscope the presence of cracks in the details of the units of tractors UMZ-8040.2 with different operating periods was estimated. The defectoscopic control of more than 500 parts of various applications was carried out in 50 tractors that were in service until 17 years since the date of issue in agricultural enterprises of the Kiev and Chernihiv regions of Ukraine. Defectoscopic inspection was carried out during the defect of the details of these tractors received for repair.

The dependence of the kinetics of the accumulation of cracks on the surface of the details of the mechanical systems (knots) of the tractor proposed in the work is exponential within the studied range of operating life and is determined in terms of the fractal dimension of the surface. A dimensionless index of the fractal dimension of micro-plastic strain amplitudes, which is a kinetic characteristic of the cumulative inelasticity in the surface layer and is defined by the Hurst parameter $H$ by the below formula, was chosen as a parameter characterizing the metal damage:

$$
\frac{R(t)}{S(t)}=\frac{X(t) \max -X(t) \min }{S(t)},
$$

where $R / S$ - normalized range;

$R$ - deviation range $X$;

$X(t)$ max - maximum value for $X$;

$X(t) \min$ - minimum value for $X$.

The range is cumulative deviation over $N$ periods or the difference between the maximum and minimum calculated values of the parameter $X$ :

$$
X=\sum_{i=1}^{t}\left(n_{i}-M_{N}\right),
$$

where $X$-cumulative deviation over $N$ periods;

$n_{i}-$ deviation during a period of loading,

$M_{N}-$ mean $n_{i}$ over $N$ periods.

The method of statistical measurement used in the work involves specifying objects of defectoscopic control in order to distinguish defective parts. The available details of the individual tractor units were divided into several categories: 
1. high-stressed components with high probability of destruction;

2. details, the technical condition of which is determined by the combined effect of force factors with aggressive environmental conditions;

3. parts, which undergo a minor damaging operation;

4. parts, in which damage is detected only visually (small, non-metallic, etc.).

Within the framework of the proposed methodological approach, the potential fracture hazard and damage to the third and fourth categories of parts: fixing, rubber, non-metal, etc. were not taken into account. In the work for the detection of cracks, a portable vibration current defectoscope was used. This defectoscope meets the requirements related to the specifics of the control of parts of tractor units. The main ones are:

- versatility in relation to the metal of the investigated parts (automatic adjustment on the metal object of control);

- information about the presence of damage in the investigated details (the smallest sizes of detected cracks: depth - more than $0.2 \mathrm{~mm}$, length - more than $3 \mathrm{~mm}$, width - more than $0.1 \mathrm{~mm}$ );

- ability to change the sensitivity (the ability to set the minimum size of defects that a defectoscope can detect);

- no need for special preparation of controlled surface of parts (the defectoscope is practically not sensitive to surface roughness and is able to detect defects in surface roughness below $60 \mu \mathrm{m}$;

- absence of the edge effect and the effect of removal of the sensor, the wear of the sensor against friction on the monitored surface;

- detection of cracks at the maximum gap between the instrument sensor and the monitored surface to $3 \mathrm{~mm}$.

The applied portable defectoscope has the ability to switch the range of sensitivity and selectivity of the device, that is, it is possible to detect cracks which sizes are larger than certain values, which is especially important for the flaw detection of the entire range of tractor parts.

Before conducting defectoscopic investigations of details and structural elements it is mandatory to check the work of the defectoscope on control samples. Control samples of cracks of different lengths were obtained on metal samples of metal alloys as a result of the propagation of fatigue cracks from the stress concentrator applied to the edge of the sample.

\section{Results and discussion}

The danger of the spread of cracks in the details and structural elements significantly depends on the size of the cross-section of the detail (structural element), where the crack originated and is developing. The methods of fracture mechanics use to calculate the degree of fracture hazard, and an approximate approach was implemented in order to isolate the details of detected cracks in certain ranges of relative length in this work. So, for example, according to this approach, the details of the steering control of tractor are considered as parts of small, medium and large size.

According to the applied gradation, small size details include the billet, swivel lever, earring, nut, ball finger, bolts terminal, lock pin, hub liner, hub, bolt, screw cover and the adjusting screw.

Parts of the average size of the steering control include: the steering column (shaft), body of hydro amplifier of the steering control, middle shaft, intermediate shaft, front shaft, steering tie rod tip, axis of adjusting screw, cardan hinges, rack, intermediate prop, splined bush, pin, right wall of the rack, worm, sector, rack, stem, and the piston. The details of the large size of the steering control include the retractable pipe, brackets, semi axis, pipe of the steering column, steering shaft and the rotary shaft.

The kinetics of the accumulation of cracks in the details of systems and assemblies of tractors of different durability is presented in Fig. 1. The coordinates of the graphs are as follows: the ordinate axis - the probability of occurrence of the critical state $P=n_{d} / N$ (the ratio of the number of cracks $n_{d}$, detected in the total number of investigated, significant in terms of the limiting condition of operation safety, details $N$ ); axis of abscissa - relative durability $D=t_{e x} / t_{\text {base }}$ (ratio of the duration of operation 
of tractors $t_{e k s}$ relative to the base operation $t_{\text {base }}$, which in the calculation is 17 years). Fig. 1 also describes the trend line equation and the accuracy of the approximation of $R^{2}$.

Scientific notation equations, as exponential functions (Fig. 1), in contrast to the linear relationship are explained by the analogy of the physical process of accumulation of scattered damage in the samples of metal during cyclic loading. The view of this analogy will continue to apply the methods of prediction of durability to failure details for evaluation of the limit technical condition tractors with an array of cracks in the details.

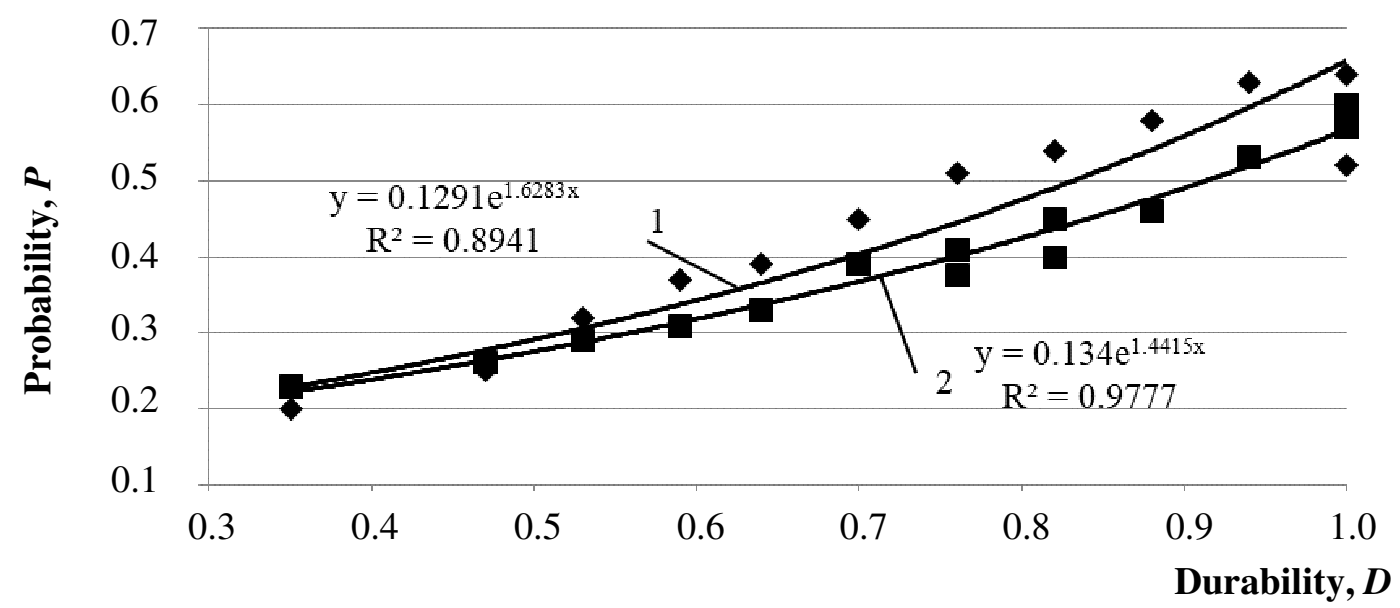

Fig. 1. Kinetics of accumulation of cracks in details of tractors of different durations of operation: 1 - steering system; 2 - hinged device of the tractor

Motorcycle hours of tractor use in agricultural enterprises during the calendar year are difficult to establish, since only one year of release of the unit is known from the operating documentation. In the given work the estimated duration of operation of tractors was obtained on the basis of the following assumptions:

- performed in the work the defectoscopic control allows with a certain degree of reliability and authenticity to detect fatigue cracks in the details of the knots of tractors involved in mechanized and transport work under the load, which led to the emergence of operational damage;

- believed that all the explored tractors of one year of issue worked practically the same hour resource;

- basic choice was the 17 -year lifetime of tractors, which is twice higher of the resources specified by the manufacturer.

The regularities considered in the work reflect the consequences of the statistical process of accumulation of an array of fatigue cracks in the details (knots) of tractors within the limits of the studied range of service life. Analysis of the statistical process of accumulation of scattered damage of structural materials under fatigue should be submitted in terms of the fractal dimension of the surface the Hurst parameter $(H)$. The characteristic of the development of the statistical process in time can be approximated with a certain degree of reliability by the exponential function (Fig. 1). The general analysis of the kinetics of the accumulation of the array of cracks in the details of all systems (nodes) of tractors (Fig. 2) allows to predict the degree of kinetics outside the studied duration of operation.

Such an a priori forecast of the characteristics of the time series of the kinetics of the accumulation of fatigue cracks indicates a likely change in the dynamics of the appearance of damaged details, which, in case of exceeding the critical parameter of unit damage, corresponds to an increase in the probability of a sudden occurrence of emergency situations in mechanized or transport jobs. The regularities of the kinetics of the accumulation of operational defects in the array of details of separate systems (nodes) of tractors are similar to the kinetics of the deformation hysteresis of the surface layer of metal structures represented by the parameter $N$. The difference lies in the different speeds of the dynamic processes of mechanical loading of laboratory samples and original parts. 
Consequently, comparing the boundary state of identical fracture processes to a single criterion creates conditions for determining the predictive parameters of the state of critical damage. Proceeding from this self-similarity of the characteristics of the processes of destruction in the time, it is advisable to set the time of the onset of the critical state at a certain probability $P$. This criterion corresponds to the degree of accumulation of operational defects, exceeding of which characterizes a significant increase in the probability of emergency situations in mechanized and transport works with the participation of tractors.

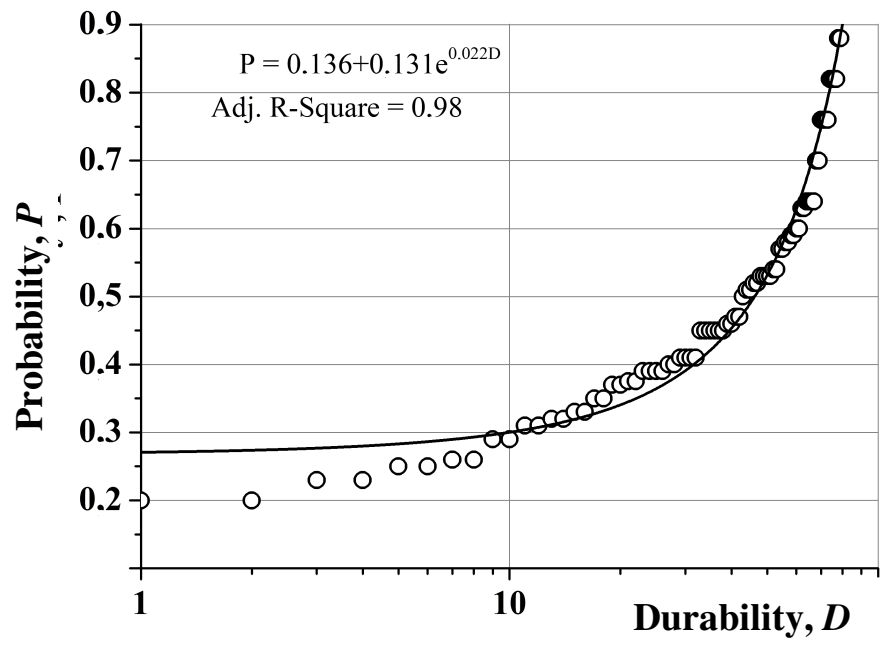

\section{Fig. 2. Kinetic characteristics of accumulation of operational damage in array of details of} tractor units on explored basis of long-term exploitation (17 years)

The relative number of details with operational cracks in each of the examined aggregates of the general ensemble of tractors is approximately the same for the whole sample of the details of each tractor. The critical durability of the tractor operation for a probability $R \approx 0.4$ is $D_{\text {crit }}=0.7-0.8$, which corresponds to 13-14 years of operation. Consequently, this indicator can serve as a criterion for discontinuation of tractor operation, the flaw detection of parts in order to detect cracks and repair (replace) the defective parts. In accordance with the Technical Rules for the safety of machines (approved by the Cabinet of Ministers of Ukraine dated January 30, 2013, No. 62) and the Technical Regulation of components and characteristics of wheeled agricultural and forestry tractors (approved by the Cabinet of Ministers of Ukraine from December 28, 2011, No. 1368) the requirements for trouble-free operation, that put forward to the technical condition of machine details is mostly qualitative (expected), instead of the introduction of quantitative indicators that could be obtained technically by means of control methods.

At present, the practice of detecting operational defects in details of tractors, self-propelled agricultural machines and cars is largely based on planiometric or organoleptic control methods, which are quite subjective. It is necessary to add to the Rules for the Technical Operation of Tractors and Mobile Agricultural Machinery the requirements of instrumental (using portable defectoscopes) detection of cracks of dangerous sizes in the details of the knots, and to consider the visual-planimetric control as not in compliance with the current requirements of this normative document. The significance of the impact of the defectoscopic control on the safety increases with the length of stay of tractors, combines and SAM in operation, in particular after 10-12 years. Introduction to the practice activities of repair units of the methodology of operative defectoscopic control and the rules for assessing the risk of exploitation of mobile agricultural machinery with defects will significantly increase the level of detection of defective agricultural aggregates annually. The use of the proposed approaches in the Rules of Technical Operation of Tractors and Mobile Agricultural Machinery is aimed at reducing the number of accidents of agricultural aggregates and misadventures.

The obtained statistical data on the existence of operational cracks can also be used to calculate the probability of occurrence of emergencies involving mobile agricultural machinery. In this calculation technique it is expedient to use data not about the linear dimensions of the detected defects, but the available signs that revealed cracks of varying degrees of danger in terms of the destruction of the detail. This allows us to estimate the tractor's malfunction in the probabilistic aspect, depending on 
the implementations of a set of signs, that is, for possible variants of the presence or absence of cracks of the boundaried and admissible sizes.

\section{Conclusions}

1. The obtained experimental characteristics of the presence of dangerous mechanical defects in the details of the tractor units allowed to determine the durability of operation, after which the probability of accumulation of critical damage accident increases significantly.

2. The necessity of changes to the Rules of technical exploitation of tractors and other mobile agricultural machinery concerning the carrying out of instrumental (with the help of portable flaw detectors) detection of cracks in dangerous sizes in details is substantiated in order to extend the term of the assigned resource as additional to the current requirements of visual-planimetric control.

3. For the investigated types of tractor units, a probabilistic criterion for the termination of the accident-free operation of the tractor was proposed and the fractoscopy of the parts was performed in order to detect and eliminate cracks by replacing or repairing defective parts. The probability criterion of $P=0.4$ corresponds to 13-14 years, the operation depending on the force impact on agricultural units.

\section{Acknowledgements}

This work was supported by a grant of the Ministry of Science and Education of Ukraine, contract _110/495/01.01.2016, Project code: UA 1649501 01, NULES Project title - "Experimental researches of parameters and modes of functioning of system of maintenance of agricultural machinery", phase 6: Experimental researches of parameters and modes of functioning of system of operational safety for agricultural aggregates.

\section{References}

[1] Ivanovs S., Bulgakov V., Nadykto V., Kuvachov V. Theoretical investigation of turning ability of two-machine sowing aggregate. Proceedings of 17th International Scientific Conference "Engineering for rural development". Jelgava, Latvia, May 23-25, 2018, Latvia University of Agriculture. Faculty of Engineering. Vol. 17, pp. 314-322.

[2] Bulgakov V., Ivanovs S., Adamchuk V., Nowak J. Theoretical investigation of steering ability of movement of asymmetric swath headerand-tractor aggregate. Proceedings of 17th International Scientific Conference "Engineering for rural development". Jelgava, Latvia, May 23-25, 2018, Latvia University of Agriculture. Faculty of Engineering. Vol. 17, pp. 301-308.

[3] Bulgakov V., Pascuzzi S., Nadykto V., Ivanovs S. A mathematical model of the plane-parallel movement of an asymmetric machine and tractor aggregate. Journal of Agricultural Engineering. Vol. 49, No 1, 2018, pp. 258-271.

[4] Bulgakov V., Nikolaenko S., Holovach I., Ivanovs S., Vartukapteinis K. Theoretical investigations of oscillations of root crop head cleaner hanged on integral row-crop tractor. Proceedings of 16th International Scientific Conference "Engineering for rural development". Jelgava, Latvia, May 24-26, 2017, Latvia University of Agriculture. Faculty of Engineering. Vol. 16, pp. 1395-1408.

[5] Yata V.K., Tiwari B.C., Ahmad, I. Nanoscience in food and agriculture: research, industries and patents. Environmental Chemistry Letters, vol. 16, 2018, pp. 79-84.

[6] Dubbini M., Pezzuolo A., De Giglio M., Gattelli M., Curzio L., Covi D., Yezekyan T., Marinello F. Last generation instrument for agriculture multispectral data collection. CIGR Journal, vol. 19, 2017, pp. 158-163.

[7] Viba J., Lavendelis E. Algorithm of synthesis of strongly non-linear mechanical systems. In Industrial Engineering - Innovation as Competitive Edge for SME, 22 April 2006. Tallinn, Estonia, pp. 95-98.

[8] Gorobets V.G., Trokhaniak V.I., Rogoskii I.L., Titova L.L., Lendiel T.I., Dudnyk A.O., Masiuk M.Yu. The numerical simulation of hydrodynamics and mass transfer processes for ventilating system effective location. INMATEH. Agricultural Engineering. Bucharest. Romania, vol. 56, No 3, 2018, pp. 185-192. Scopus. WoS. 
[9] Pinzi S., Cubero-Atienza A.J., Dorado M.P. Vibro-acoustic analysis procedures for the evaluation of the sound insulation characteristics of agricultural machinery. Journal of Sound and Vibration, vol. 266 (3), 2016, pp. 407-441.

[10] Voinalovych A.V., Motrich M.N. Control of the technical state of agricultural aggregates by facilities of fault detection. Mechanization in agriculture. Sofia. Bulgaria, Year LXI, ISSN 08619638, issue 12, 2015, pp. 29-31. 